\title{
In Wirklichkeit können wir neue sozioökonomische Realitäten imaginieren (lernen)
}

\section{Ein essayistisches Vorwort}

\section{Maja Göpel}

Menschen sind kreative und sinnsuchende Wesen. Wir lieben es, zu lernen, Rätsel zu lösen und Neues zu gestalten. Wir suchen nach Verständigung und Verständnis. Wir sind erzählende und reflektierende Wesen, die in einer Welt der Geschichten leben. Und diese Geschichten lernen wir natürlich durch unser soziales Umfeld und in unseren Bildungsinstitutionen. Sie wirken auf mindestens drei Ebenen: Als Individuum entwickelt jeder Mensch eine persönliche Sicht auf die Dinge, anhand derer wir Urteile und Entscheidungen treffen. Als soziale Wesen verwenden wir normative Kodizes und einen kanonisierten Alltagsverstand, um diese Entscheidungen gegenüber anderen zu vertreten und sich in Beziehung zu ihnen zu setzen. Im politischen Raum schließlich herrschen die großen gesellschaftlichen Erzählungen und Paradigmen, die als Referenzrahmen für angemessenes oder auch rationales Handeln gelten und damit auch das Gefühl einer Schicksalsgemeinschaft kreieren. Daher wirken die Geschichten auf den drei Ebenen auch immer aufeinander ein. Und der gigantische Entwicklungsfortschritt der Spezies Mensch liegt nicht zuletzt genau darin begründet, dass dadurch koordinierte Routinen, ausdifferenzierte Arbeitsteilung, komplexe Institutionen und bahnbrechende Innovationen über eine erzählerische Begründung ihrer Sinnhaftigkeit funktionieren. Allein deshalb kann die Bedeutung des Hinterfragens und Neuausrichtens der vielleicht zentralen Geschichte des 19. und vor allem 20. Jahrhunderts gar nicht überschätzt werden: die Gesellschaft des (grenzenlosen) monetären Wachstums.

M. Göpel ( $\square)$

Wissenschaftlicher Beirat der Bundesregierung Globale Umweltveränderungen

(WBGU), Berlin, Deutschland

E-Mail: mgoepel@wbgu.de

(C) Der/die Autor(en) 2021

J. Urban et al. (Hrsg.), Wirtschaft neu lehren, Sozioökonomische Bildung und Wissenschaft, https://doi.org/10.1007/978-3-658-30920-6_2 
Ihr zugrunde lag und liegt eine extraktivistische Perspektive auf unsere Umwelt sowie auf Menschen und ihre Arbeitskraft. Technologien wurden und werden häufig dafür ersonnen, noch mehr aus diesen beiden „Produktionsfaktoren" herauszuholen. Die Überzeugungsleistung dieser Perspektive kann nur dann verstanden werden, wenn wir den Kontext ihres Ursprungs mit in das Blickfeld nehmen. Dieser lag in einer „leeren Welt“ (Daly 2015) mit etwa einer Milliarde Menschen, wenigen Maschinen und scheinbar endlos verfügbarer Natur. Doch das 17. und 18. Jahrhundert ist nicht zu vergleichen mit einer „,vollen Welt" (ebd.), in der nun schon fast acht Milliarden Menschen leben, der Pro-Kopf-Konsum sich bei gleichbleibender Ungleichverteilung vervielfacht hat und sogenannte „entwickelte Gesellschaften“ sich durch eine Kohabitation mit unzähligen Maschinen und einer zunehmenden Anzahl digitaler Assistenzen auszeichnen. Dennoch hält die Wachstumserzählung an, ja es scheint fast unmöglich, sie nüchtern, empirisch und damit auch kritisch zu betrachten, ohne in eine kommunistische oder sozialistische Ecke gestellt zu werden. Das ist vielleicht weniger erstaunlich, wenn wir uns klarmachen - so wie es Politökonominnen und Politökonomen auch immer tun -, dass mit jeder hegemonialen Geschichte und jedem Weltbild natürlich auch Interessen und Privilegien verknüpft sind. Und gerade die Geschichte des grenzenlosen Wachstums erlaubt es, die Verteilungsfrage zu umgehen: so lange es immer mehr von allem geben wird, können aktuelle Ungleichverteilungen als Übergangszustände gelten. Heute jedoch sind die Dokumentationen von planetaren Veränderungen und von langfristiger, struktureller Ungleichheit so erfahrbar geworden, dass die sinnschaffende und Legitimation stiftende Wirkung der Erzählung des unendlichen Wachstums immer mehr verloren geht. Eine immer weiter infrage gestellte Geschichte verliert zunehmend ihre Sinnhaftigkeit, ihre Bindung und Kooperation vermittelnde Funktion.

Auf den drei Ebenen betrachtet würden Psychologinnen und Psychologen also von einer zunehmenden kognitiven Dissonanz sprechen; Sozialwissenschaftlerinnen und Sozialwissenschaftler würden einen Vertrauensverlust diagnostizieren, durch den das eher unhinterfragte Funktionieren einer Gemeinschaft und ihrer Institutionen ins Wanken gerät. Wir können dies in vielen Ländern beobachten. Die politökonomische Transformationsforschung widmet sich der Ebene großer gesellschaftlicher Erzählungen und beschreibt diese Zeiten als strukturelle Krise: die etablierten Prozesse der Produktion und des Konsums sind mit zunehmenden Herausforderungen konfrontiert. Die Wirtschaft steht vor dem Problem schwindender Ressourcen und abnehmender Regenerationsfähigkeit der Ökosysteme. Verschärft wird diese krisenhafte Situation durch ein zunehmend volatiles und fragiles Finanzsystem, das die 
bereits bestehenden Ungleichheitstendenzen erheblich verstärkt. Obendrein bricht sich eine Digitalisierungsagenda Bahn, durch die gigantische Konzerne soziale Sicherungssysteme, Marktstrukturen und Steuermodelle in ungekanntem Ausmaß disruptieren und neu konfigurieren. Daher schwindet auch der Glaube rapide, dass diese Herausforderungen mit ein paar optimierenden Anpassungen in den Griff zu bekommen sind. Was bisher als normal, unhinterfragt und gegeben akzeptiert wurde, kommt auf den Prüfstand. Die Rufe nach radikalen anstatt inkrementellen Veränderungen werden häufiger - und die nach einem Zurückdrehen der Geschichte womöglich auch. Das bestehende System ist im Stress.

Doch aus der Perspektive der Transformationsforschung ist eine solche fundamentale Infragestellung die Voraussetzung für radikalen Wandel. Denn der Vorstellungsraum von dem, was sich verändern muss, schlägt vom Ausder-Box-Denken in ein Über-die-Box-Denken um. Die Box selbst braucht eine andere Form. Und damit bekommen alternative, plurale und sozioökonomische Ansätze in der Forschung und Lehre eine ganz andere Chance, aus der Nische in die Normalität vorzudringen. Denn obgleich unsere Welt dinghaft daherkommt, so ist sie doch - wie Ökologie und konstruktivistische Sozialwissenschaft schon lange formulieren - ein fortlaufender Reproduktionsprozess von Leben und Zusammenleben. Dafür gehen wir Beziehungen ein und schaffen Prozesse, die sich dann in ihrer Gesamtheit als sich selbst stabilisierende Systeme oder Strukturen manifestieren. Das ist einerseits praktisch, weil vieles unhinterfragt und aufeinander abgestimmt läuft. Andererseits entstehen dadurch genau die Pfadabhängigkeiten, die es so schwer machen, transformative Veränderungen in die Tat umzusetzen. Denn das, was wir Realität nennen (von lateinisch res: Ding, Sache), beeinflusst natürlich die Sinnsuche, Imagination und Wissensbestände der diese Realität reproduzierenden Menschen. Unsere Freiheit ist immer von den Rahmenbedingungen, in denen wir leben, beeinflusst. Karl Marx und Friedrich Engels 1972 [1852] haben das in pessimistischer Perspektive auf den Punkt gebracht: „Die Tradition aller toten Geschlechter lastet wie ein Alp auf dem Gehirne der Lebenden“ (ebd., S. 115). Auf die heutige Situation angewandt beschreibt auch das Büro für Technikfolgen-Abschätzung des Deutschen Bundestages (TAB) die prägende Kraft der Gegenwart auf die Zukunft: Visionen, Erzählungen, Szenarien, Simulationen etc. haben zwar ,einen Inhalt in Form von Vorstellungen über zukünftige Entwicklungen, beruhen jedoch ausschließlich auf gegenwärtigen ,Inputdaten' wie Wissen, Interessen, Annahmen und Werten. Wie das erste mit dem zweiten zusammenhängt, ist meist nicht transparent“ (Grunwald 2012, S. 26, Hervorhebung im Original). Ein Blick in die HightechStrategien der letzten Jahrzehnte zeigt deutlich, welche Vorstellungen über 
zukünftige Entwicklungen hier dominant waren und wie einflussreich das weitgehend unhinterfragte Modell grenzenlosen monetären Wachstums dort war. Sozioökonomische Ansätze, analog der reflexiven Transformationsforschung, sind also deshalb so wichtig, weil sie nicht eine vermeintlich objektive Realität fortschreiben, sondern sich der Effekte von Wirklichkeit (von actus, lateinisch: Taten) und erzählerischer Verarbeitung von Wirklichkeit bewusst annehmen: „Eine Transition ist ein Prozess des fundamentalen und irreversiblen Wandels der Kultur, der (institutionellen) Strukturen und Praktiken einer Gesellschaft. [...] Transitionen sind das Ergebnis einer Ko-Evolution von ökonomischen, kulturellen, technologischen, ökologischen und institutionellen Entwicklungen auf unterschiedlichen Ebenen“ (DRIFT 2017).

Allein aus dieser Perspektive ist der Auftrag für Universitäten und Bildungsinstitutionen gerade in Transformationszeiten ein sehr gesellschaftlicher. Der Begriff der „Transform-Abilität“ fasst dies zusammen und wurde von einem Wissenschaftlerteam um Frances Westley definiert als „die Fähigkeit, unbekannte Anfänge zu kreieren, aus denen sich eine völlig neue Form des Lebens entwickelt, wenn die gegebenen ökologischen, ökonomischen und sozialen Voraussetzungen das aktuelle System unhaltbar machen“" (Westley et al. 2011, S. 763). Club of Rome-Vordenker Erich Jantsch (1970) hat daher bereits vor fünfzig Jahren formuliert, dass der Auftrag der Universität sei, die Veränderungsfähigkeit einer Gesellschaft zu erhalten. Sein Buch zur „Selbstorganisation des Universums“" (Jantsch 1979) hatte das Ziel, Voraussetzungen und Rahmenbedingungen zu finden, unter denen komplexe und lebensfähige Systeme entstehen. In seinem Universitätsmodell war der transdisziplinäre Anteil wesentlich größer als die Grundlagenforschung - und dem Auswendiglernen von hoch reduktiven und geschlossenen Modellen zur Anwendung auf eine sich ständig in Veränderung befindlichen Welt hätte er wohl kaum einen Platz in der Universität eingeräumt. Denn die Fähigkeit, unbekannte Anfänge und damit eine sich selbst neu organisierende Wirklichkeit zu kreieren, hängt von den Menschen ab, die daran beteiligt sind und beobachten, wann ein System seine Tragfähigkeit zu verlieren droht sowie sich entsprechende unbekannte Anfänge ausdenken und mit ihnen experimentieren. Sozio-kulturelle Anteile von Transform-Abilität umfassen also einen fortlaufenden Lernprozess, um in der gesellschaftlichen Geschichtsschreibung Tunnelblicke zu vermeiden und Verfallsdaten von guten Ideen rechtzeitig zu identifizieren. Entsprechend respektiert und gefördert können so Frühwarnsysteme entstehen und der Möglichkeitsraum für zukünftige Gestaltung von gesellschaftlicher Entwicklung wird systematisch weit gehalten. Nach 45 Jahren nicht ausreichend transformativer Nachhaltigkeitsagenda liegt hier ein riesiges Potenzial menschlicher Emanzipation und Evolution. 
Dankenswerterweise hat die Wissenschaft des 21. Jahrhunderts bereits Vieles im Köcher, um den ökonomistischen Tunnelblick auf menschliche Entwicklung zu ersetzen. Soziologie, heterodoxe Ökonomik, positive Psychologie, Glücksforschung und Neurowissenschaften stellen Grundannahmen zu ,repräsentativen Akteuren" orthodoxer wirtschaftswissenschaftlicher Modelle ebenso infrage wie die Ökologie, Quantenphysik und Forschung zu Erdsystemen und komplexen Systemen. Sie zeichnen ein regeneratives Bild von Mensch-Mensch-NaturBeziehungen, das eine radikale Alternative zur mechanistischen Akkumulation von Dingen und Geldwerten bietet. Einer ihrer wichtigen gemeinsamen Nenner ist die systemische Betrachtung der Welt, in der Elemente und ihre Qualität nicht ohne den Kontext und die Verbindungen, in die sie eingebettet sind, verstanden werden können. Auch können diese Verbindungen nicht einfach gelöst und einzelne Elemente ersetzt werden, ohne dass sich die Dynamik des Systems verändert: „Naturkapital“ ist weit mehr als eine Ansammlung von Ressourcen, die frei ab- und aufgebaut werden können. Resiliente Strukturen der Wertschöpfung brauchen ein systemisches Verständnis von Effizienz und nicht eines, das einzelne Prozesse auf maximalen Output und damit auch auf maximale Fragilität trimmt. Beschleunigung und Vermehrung von Abläufen und Erträgen sind nicht per se positive Attribute, sondern mögliche Ziele für eine optimale Entwicklung von Systemen, die aber auch herunterreguliert werden sollten, wenn sie den regenerativen Zyklen nicht mehr entsprechen und die Substanz zu stark schwächen. Die Glückforschung zeigt auf, dass neben den überlebensnotwendigen Grundbedürfnissen vor allem der relative Wohlstand im Verhältnis zu anderen, das Gefühl der Teilhabe an Gesellschaft und die Gestaltbarkeit des eigenen Lebens zur Zufriedenheit beitragen. Zugleich weisen Studien nach, dass sich Gesellschaften mit weniger Ungleichheit durch bessere Gesundheitswerte und weniger Kriminalität auszeichnen (siehe eine Zusammenfassung dieser Befunde in Göpel 2016).

Diese systemischen Betrachtungen von menschlicher und natürlicher Entwicklung rücken das Verhältnis von Zielen und Mitteln wieder zurecht. In der Gesellschaft des monetären Wachstums sind diese wichtigen Unterscheidungen im Laufe ihrer Geschichte verloren gegangen und mehr von allem war auch gleich besser für alle. Mittel sind dabei oft $\mathrm{zu}$ Zielen verkommen: das BIPWachstum ist das inzwischen wohl prominenteste Beispiel, aber auch endlose Produktivitätssteigerungen oder kontinuierliche Erhöhung der Wettbewerbsfähigkeit gelten heute als sakrosankte Ziele für gelingende Entwicklung, ohne sie qualitativ auf den Prüfstand zu stellen oder die Effekte ihrer Steigerung auf das Wohlergehen von Mensch und Natur wirklich sachgerecht zu bilanzieren. Laut Golding und Kutarna (2016) und ihrem Buch Die Zweite Renaissance besteht 
also ,der erste mutige Akt“ darin, ,eine langfristige Perspektive zu verfolgen, die sich auf das übergeordnete Gesamtbild konzentriert" (ebd., S. 359). Ihnen zufolge helfen die Tugenden Ehrlichkeit, Wagemut und Würde (ebd.), denn „die gebildeten Menschen der ersten Renaissance veränderten in Anpassung an die neuen Herausforderungen ihre geistige Landkarte der Welt, und zwar vollkommen“ (ebd., S. 351). Es ist also keineswegs ein negatives Label, als „utopisch“, ,unrealistisch“ oder ,,idealistisch“ bezeichnet zu werden - im Gegenteil. Die Verfechterinnen und Verfechter einer sich evolutionär entwickelnden Wirklichkeit haben den Vorteil, dass sie neben den wahrscheinlichen und plausiblen Zukunftsentwicklungen auch noch die wünschenswerten denken und vorbereiten können.

Viel mehr Sinn kann ein Bildungsideal nicht $\mathrm{zu}$ vermitteln versuchen weniger sollte es aber in transformativen Zeiten wie den heutigen auch nicht sein.

\section{Literatur}

Daly, H. (2015). Economics for a Full World. Essay for the Great Transition Initiative. Tellus Institute: Cambridge (USA). https://greattransition.org/publication/economicsfor-a-full-world. Zugegriffen: 06. März 2020.

DRIFT (Dutch Research Institute for Transitions) (2017). Transitions. Rotterdam: Erasmus Universität. https://drift.eur.nl/about/transitions/. Zugegriffen: 07. Dez. 2017.

Göpel, M. (2016). The Great Mindshift. How a New Economic Paradigm and Sustainability Transformations Go Hand in Hand. Heidelberg: Springer.

Golding, I., \& Kutarna, C. (2016). Die Zweite Renaissance. Warum die Menschheit vor dem Wendepunkt steht. München: Finanzbuchverlag (FBV).

Grunwald, A. (Hrsg.) (2012). Technikzukünfte als Medium von Zukunftsdebatten und Technikgestaltung. In Karlsruher Studien Technik und Kultur 6. Karlsruhe: KIT Scientific Publishing.

Jantsch, E. (1970). Inter- and Transdisciplinary University: A systems approach to education and innovation. Policy Sciences, 1(4), 403-428.

Jantsch, E. (1979). Die Selbstorganisation des Universums: Vom Urknall zum menschlichen Geist. München: Hanser.

Marx, K., \& Engels, F. (1972). Der achtzehnte Brumaire des Louis Bonaparte. In K. Marx \& F. Engels. Werke, Bd. 8 (S. 115-123). Berlin (DDR): Dietz.

Westley, F., Olsson, P., Folke, C., Homer-Dixon, T., Vredenburg, H., Loorbach, D., Thompson, J., Nilsson, M., Lambin, E., Sendzimir, J., Banerjee, B., Galaz, V., \& van der Leeuw, S. (2011). Tipping toward sustainability: Emerging Pathways of transformation. Ambio, 40(7), 762-780. 
Open Access Dieses Kapitel wird unter der Creative Commons Namensnennung 4.0 International Lizenz (http://creativecommons.org/licenses/by/4.0/deed.de) veröffentlicht, welche die Nutzung, Vervielfältigung, Bearbeitung, Verbreitung und Wiedergabe in jeglichem Medium und Format erlaubt, sofern Sie den/die ursprünglichen Autor(en) und die Quelle ordnungsgemäß nennen, einen Link zur Creative Commons Lizenz beifügen und angeben, ob Änderungen vorgenommen wurden.

Die in diesem Kapitel enthaltenen Bilder und sonstiges Drittmaterial unterliegen ebenfalls der genannten Creative Commons Lizenz, sofern sich aus der Abbildungslegende nichts anderes ergibt. Sofern das betreffende Material nicht unter der genannten Creative Commons Lizenz steht und die betreffende Handlung nicht nach gesetzlichen Vorschriften erlaubt ist, ist für die oben aufgeführten Weiterverwendungen des Materials die Einwilligung des jeweiligen Rechteinhabers einzuholen.

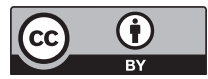

